\title{
HUMAN SARCOCYSTOSIS, AN UNCOMMON ETIOLOGY FOR MOTOR NEURON DISEASE - A RARE INTERESTING CASE REPORT
}

\author{
ANURADHA S. * AND BHARATHI K. \\ Department of Pathology, Sri Satya Sai Medical College and Research Institute, Ammapettai-603108, Tamilnadu, India. \\ ${ }^{*}$ Corresponding Author: Email- bharathi.pathos@gmail.com, sanudoc50@yahoo.co.in
}

Received: April 30, 2012; Accepted: May 24, 2012

\begin{abstract}
A 45 year old male, non vegetarian by diet, loader by occupation, presented with complaints of progressive muscle wasting and asymmetric weakness of the upper limbs of one month duration, which was followed by similar signs and symptoms in the lower limbs. There was decreased power in the affected limbs. He occasionally had muscle fasciculations. The sensory, bladder, bowel and cognitive functions were well preserved. There was no difficulty in breathing, speaking and swallowing. On the basis of history and neurological examination, a diagnosis of Motor Neuron Disease was made. All the basic investigations were found to be normal. For a definitive diagnosis, muscle biopsy was taken from various sites and to our surprise, it showed many sarcocysts filled with banana shaped bradyzoites of Sarcocystis species. Genus Sarcocystis belongs to the phylum apicomplexa and the class Coccidiea. Muscular sarcocystosis is so rare in India. Sarcocystosis presenting like a Motor Neuron Disease is a diagnostic challenge to the practitioners. The case is presented here for its rarity and to create awareness about the Sarcocystis infection and its presentation like a Motor Neuron Disease.
\end{abstract}

Key words- Human Sarcocystis Infection, Muscle Wasting, Muscle Weakness, Motor Neuron Disease, India.

Citation: Anuradha S. and Bharathi K. (2012) Human Sarcocystosis, an Uncommon Etiology for Motor Neuron Disease - A Rare Interesting Case Report. International Journal of Medical and Clinical Research, ISSN:0976-5530 \& E-ISSN:0976-5549, Volume 3, Issue 4, pp.-161163.

Copyright: Copyright@2012 Anuradha S. and Bharathi K. This is an open-access article distributed under the terms of the Creative Commons Attribution License, which permits unrestricted use, distribution, and reproduction in any medium, provided the original author and source are credited.

\section{Introduction}

Human Muscular Sarcocystosis, a rare zoonotic infection caused by an intracellular coccidian protozoa is seen in tropical and sub tropical countries[1]. Most cases reported are from South East Asia and Asia possibly reflecting the food habits, environment and sanitation levels $[2,3]$. Human infection is considered rare with less than hundred of published cases, which is very minimal when compared with the global burden of the disease. Sarcocystosis in man presents as two distinct types of clinical presentations like Intestinal Sarcocystosis and Muscular Sarcocystosis. Muscular Sarcocystosis is so uncommon where man serves as the intermediate host, who acquires the infection by ingestion of food and water contaminated by the sporocysts that are shed in the feces of the definitive hosts [4]. Close contact with carnivorous animals leads to the spread of the disease among human beings. Muscu- lar Sarcocystosis presenting like a Motor Neuron Disease offered a diagnostic challenge to us which will be described below in the case history.

\section{Case Report}

A 45 year old male, not vegetarian, loader by occupation, presented with complaints of progressive muscle wasting and asymmetric weakness of the upper limbs of one month duration, which was followed by similar signs and symptoms in the lower limbs of one month duration. There was decreased power in the affected limbs. He occasionally had muscular fasciculations. The sensory, bladder, bowel and cognitive functions were intact. There was no difficulty in breathing, speaking and swallowing. All the basic laboratory investigations done were within normal limits except that Erythrocyte sedimentation rate and creatine kinase levels were 
slightly elevated. A differential diagnosis of Motor Neuron Disease, Cervical Spondylosis, Muscular Dystrophy, Cervical Rib, Spinal Radiculitis, Peripheral Neuropathies, and Spinal Cord Tumours were considered. On the basis of history, investigations and neurological examination, a diagnosis of Motor Neuron Disease was made.

Finally muscle biopsy was done from the muscles like biceps, deltoid of the right upper limb, Microscopic examination of the tissue sections of muscle biopsy showed multiple sarcocysts filled with bradyzoites of Sarcocystis species. Fig (1), Fig (2). The muscle fibres were angulated and atrophic, Fig (3) with occassional inflammatory cell infilteration around the muscle fibres. Then with the consent of the patient, multiple biopsies were taken from biceps \& deltoid of left upper arm, gastronemius and gluteus maximus of both lower limbs. The histopathology examination showed the similar picture. Hence the diagnosis of Human Muscular Sarcocystosis was made.

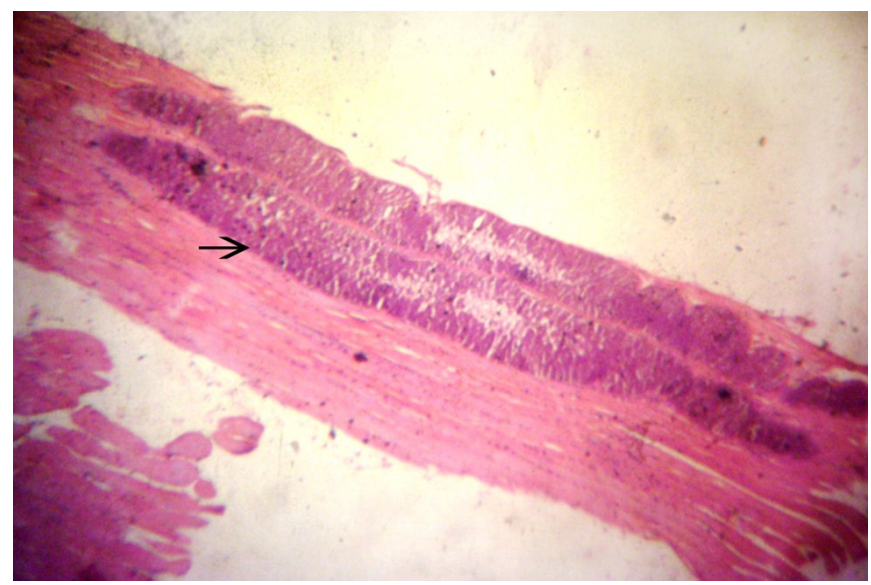

Fig. 1- Photomicrograph shows the high power view of the tangential cut sections of Gastronemius with thread like sarcocyst (arrow) filled with bradyzoites [H\&E stain -400X].

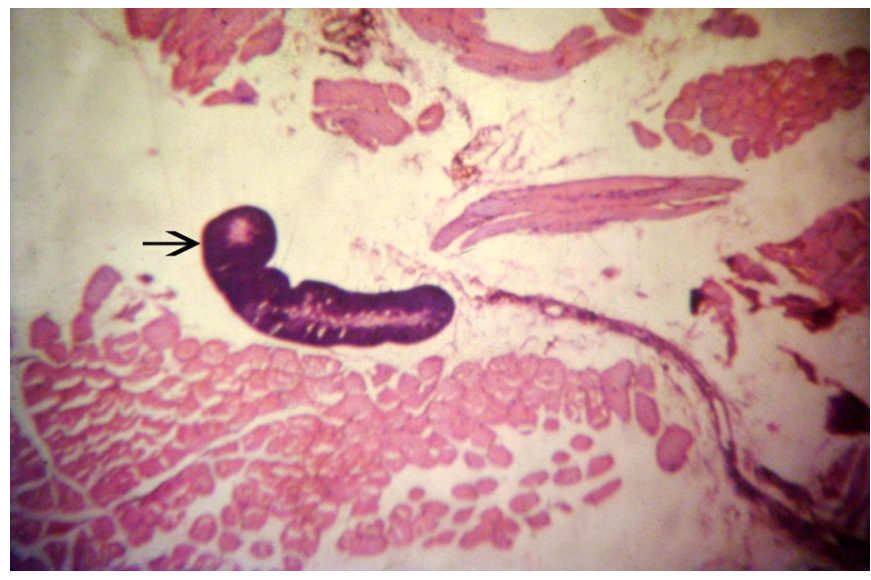

Fig. 2- Photomicrograph shows the low power view of spindle to cylindrically shaped sarcocysts (arrow) in the biceps muscle of right arm [H\&E stain -100X].

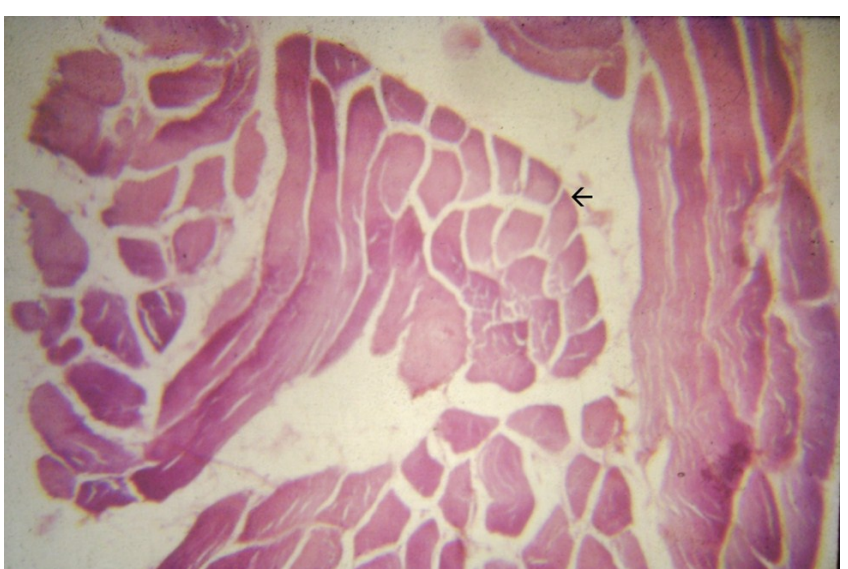

Fig. 3- Photomicrograph shows the angulated muscle fibres in sarcocystosis [H\&E stain $-100 X]$.

\section{Discussion}

Sarcocystis, apicomplexan parasite which belongs to the Order: Coccidia has an obligatory two-host life cycle. Sarcocystis spp. infection can manifest as intestinal sarcocystosis and as the rarer muscular sarcocystosis.

Intestinal sarcocystosis is caused by two species with a similar life cycle, Sarcocystis hominis and Sarcocystis suihominis. Man is the definitive host. Infection usually occurs through ingestion of undercooked meat. Sexual reproduction takes place in the intestine of definitive hosts where the oocyst matures into gametocytes. Infective oocysts and sporocysts are shed in the stools and the clinical manifestations are nausea, abdominal pain, vomiting, and diarrhea [5].

Muscular Sarcocystosis: is caused by an undetermined number of unidentified species known collectively as Sarcocystis spp. Man is the intermediate host here. The organism was first recognized in a mouse by Meischer in 1843 as white thread like cysts in the striated muscle. The first report of human infection was by Lindemanni in 1868 [6].

This species was named as 'Sarcosystis lindemanni' by Rivolta after Lindemanni in 1898. The name was proposed initially to all the intramuscular sarcocysts in humans but it was not clearly described and many species which are morphologically different were seen to cause the similar disease in every case reported, so the name is considered to be a 'nomen nudum'( a name without a recognized species) [7]. Now they were collectively called as 'Sarcocystis species'.

The life cycle of Sarcocystis species is different. Man is the intermediate host. Man acquires the infection by ingestion of food contaminated with sporocysts excreted in the faeces of carnivorous animals which are the definitive hosts of the parasite. In the intestine of man, the sporocysts release the sporozoites which then enter the lumen of the arteries. In their vascular endothelium, the sporozoites develop into schizonts which undergoes a change as first, second and three generation merozoites. This third generation of merozoites then enter into the skeletal muscle and form sarcocysts. Inside the Sarcocysts, the unicellular metrozoite multiply through asexual reproduction and then mature into crescent shaped bradyzoites. Thus the intermediate hosts which harbor the sarcocysts in their skeletal and cardiac muscles present with muscular sarcocystosis. The life cycle is completed only when the 
carnivores ingest the flesh with sarcocysts. Man is the dead end here $[7,8]$.

Muscular sarcocysts are usually asymptomatic, but they can present with muscular pain, muscular weakness, muscle wasting, lumps and focal myositis. Inflammatory reaction can be seen around the muscle fibres resulting in the atophy and angulation of muscle fibres. [Fig 3] The reason for the inflammation can be due to the rupture of the cysts of parasitized muscle fibres resulting in the release of 'sarcocystin' a toxin. Sarcocystin not only produces inflammation but may also cause weakness and pain the musles [9]. The mechanism for Motor Neuron Disease is said to be the interference of conduction at the level of motor neuron plate by the toxin. Some cases of muscular sarcocystosis reported from Malaysia were also associated with malignancies, especially of the tongue and nasopharynx [1]. Two cases from India presented with with discharging sinuses [10].

Sarcocysts are identifiable through tissue sections of muscle biopsies stained with Hematoxylin \& Eosin and PTAH stain. Sarcocystis spp can be seen in active skeletal muscle fibres and cardiac muscle [11]. Cysts are spindle to cylindrical in shape with size ranging from $50 \mu \mathrm{m}-200 \mu \mathrm{m}$ surrounded by a thin hyalinized wall. The cysts contain many banana shaped bradyzoites of size ranging from $1-3 \mu \mathrm{m}$. Other findings that can be seen in the muscle biopsy include myonecrosis, perivascular and interstitial inflammation, vasculitis and eosinophilic myositis [12].

Human muscular sarcocystosis is so rare in India with a few cases being reported across a wide range of age group [13]. Parija 2002 have described 7 cases of sarcocystosis reported from various parts of the India [14].

Diagnosis of human muscular sarcocystosis depends on the demonstration of sarcocysts in the skeletal muscle or cardiac muscle at biopsy or autopsy. Stool examination in case of muscular sarcocystosis will be negative for oocysts or sporocysts. The indirect fluorescent antibody test or ELISA can also be done but not so useful in case of muscular sarcocystosis. There is no specific therapy available for muscular sarcocystosis [15].

Infection can be prevented by protecting the food, soil and water from contaminants like human and animal feces. Thorough washing of raw vegetable for salads and cooking the meat well before consumption is essential to control the spread of muscular sarcocystosis. Alternatively freezing the meat at -5 degree Celsius for 2 days will render it non infectious.

\section{Conclusion}

Human Muscular Sarcocystosis, a rare zoonotic infection in India, caused by a coccidian protozoa belonging to the group of yet unknown species collectively called as 'Sarcocystis' species. The parasitosis can occasionally present like a Motor Neuron Disease and it can be considered in the differential diagnosis of Motor Neuron Disease. The best possible preventive measures suggested are washing the fresh vegetables thoroughly before ingestion, cooking the meat well and boiling the drinking water to kill the sporocysts.

\author{
List of Abbreviations \\ H\&E - Hematoxylin \& Eosin Stain \\ PTAH - Phospho Tungstic Acid Hematoxylin Stain \\ ELISA - Enzyme Linked Immunosorbent Assay
}

\section{References}

[1] Pathmanathan R., Kan S.P. (1992) Trop Geogr. Med., 44(1-2), 102-8.

[2] Kan S.P., Pathmanathan R. (1991) Southeast Asian J Trop Med Public Health, 22, 129-4

[3] Wong K.T., Pathmanathan R. (1992) Trans R. Soc. Trop. Med. Hyg., 86(6), 631-2.

[4] Mehrotra R., Bisht D., Singh P.A., et al. (1996) Pathology, 28 (3), 281-2.

[5] Tungtrongchitr A., Chiworaporn C., Praewanich R., et al. (2007) Southeast Asian J. Trop. Med. Public Health, 38(2), 232.

[6] Thomas J.A. (1976) J. Postgrad Med., 22, 185-90.

[7] Faust E.C., Russell P.F. (1964) Clinical Parasitology, 7th ed, Philadelphia: Lea and Febiger, 282.

[8] Fayer R. (2004) Clin. Microbiol. Rev., 17(4), 894-2.

[9] Markus M.B., Killick-Kendrick R., Garnham P.C.C. (1974) J. Trop. Med. Hyg., 77, 248-9.

[10]Agarwal P., Srivastava A. (1983) Histopathology, 7, 783-7.

[11]Gilmore H.R., Kean B.H., Posey F.M. (1942) Am. J. Trop. Med., 22, 121-5.

[12]Arness M.K., Brown J.D., Dubey J.P., Neafie R.C., et al. (1999) Am. J. Trop. Med. Hyg. 61, 548-3.

[13]Vasudevan A. (1927) Indian J. Med. Res. 15, 141-2.

[14]Subash Chandra Parija. Coccidia (2006) Textbook of Medical Parasitology- Protozoology and Helminthology, 3rd ed. 181-2.

[15]Peter F. Weller (2005) Protozoal intestinal infections and Trichomoniasis., Harrison's Principles of Internal Medicine, 16th ed. McGraw Hill companies.Inc., 1252. 